\title{
Admittance Control of Four-link Bionic Knee Exoskeleton with Inertia Compensation
}

\author{
Fei LI, Qingdong WANG, Yao XIE, Hualong XIE
}

\begin{abstract}
This paper proposes a control algorithm based on the admittance principle for the motion of the four-link bionic knee exoskeleton. Firstly, the interaction between the operator and the exoskeleton was converted into the desired trajectory of the exoskeleton. Then, the inertia compensation is achieved in light of the admittance features of exoskeleton movement. Finally, the validity of the admittance control method for four-link bionic knee was confirmed through simulation experiment. The simulation results show that the relative error of the joint angle between the operator and the exoskeleton was less than $5 \%$ at normal swinging frequency, and the interaction force between the manipulator and the exoskeleton was within $\pm 0.5 \mathrm{~N}$. The research findings lay a theoretical basis for practical application of exoskeletons.
\end{abstract}

Keywords: admittance control; exoskeleton; four-link bionic knee; inertia compensation

\section{INTRODUCTION}

Recent years have seen the proliferation of exoskeletons in military, medical aid and disability rehabilitation, etc. As a bionic mechanical system, the exoskeleton can assist human movement by enhancing the operator's limb strength, making it more energy-efficient to move articulations. With the aid of exoskeleton, it is possible for a disabled operator to achieve normal movement [1-3]. The key to exoskeleton operation lies in the operator's manipulation of the tool and the coordinated movement between the operator and the tool.

Most of exoskeletons adopt a unilateral knee, i.e. a knee with only one degree of freedom (DOF), despite the complex motions of human knee, namely, rolling and sliding [4-6]. After all, the unilateral knee is much simpler and easier to achieve precise control than the four-link bionic knee. However, many scholars [7, 8] found that the unilateral knee cannot simulate the instantaneous changes in human knee movement, and may cause gait difference between the operator and the tool.

Many new exoskeletons have been developed to solve the above problem. For example, the University of California, Berkeley designed a unilateral knee exoskeleton controlled by sensitivity amplification $[9,10]$. Based on the exoskeleton dynamics model, the control method can capture and magnify the operator's intention, such that the exoskeleton can move coordinately with the operator. Japan's Tsukuba University put forward the Hybrid Assistive Limb (HAL). The exoskeleton relies on the electromyogram (EMG) signals to identify the operator's intention, recognize the joint torque, and realize coordination with the operator's movement [11, 12]. However, the detection of EMG signals brings new problems like wear, noise and interference.

Northwestern University tested the control of unilateral knee exoskeleton mechanical leg based on admittance principle, revealing that the admittance control can effectively increase the flexibility of the exoskeleton mechanical leg [13]. Extended from impedance control, the admittance control is achieved through a force outer ring and a position inner loop [14]. In this method, the operatortool interaction is transformed into the desired trajectory of the exoskeleton by the admittance model.

Currently, the exoskeleton is only suitable for people with both legs, which cannot meet the needs of above-knee amputees to realize both daily walking and assisted walking functions. In this paper, an asymmetric exoskeleton for above-knee amputees is presented and the admittance control study of its four-link bionic knee is focused, which is more complex and harder to control than the uniaxial knee. In addition, the center of instantaneous change of the target exoskeleton exhibits as a J-shaped curve $[15,16]$. Specifically, the admittance model for the four-link bionic knee was established by the equivalent method, and used to transform the operator-tool interaction into the desired trajectory of the exoskeleton. Then, the validity of the admittance control method for four-link bionic knee was confirmed through simulation experiment, laying a theoretical basis for practical application.

\section{DESIGN OF FOUR-LINK BIONIC KNEE EXOSKELETON}

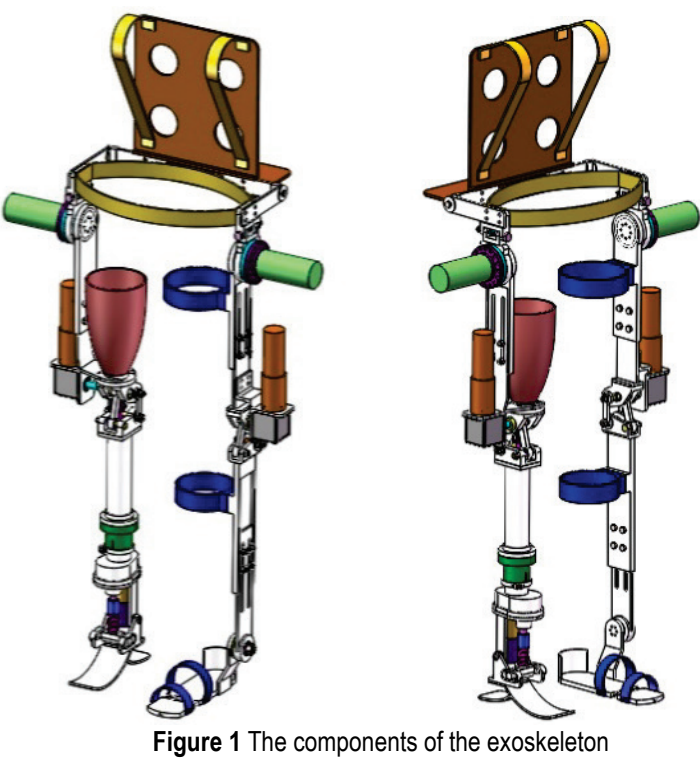

As shown in Fig. 1, the proposed asymmetric exoskeleton consists of the lumbar support, hip joint, thigh, bionic knee, calf and ankle joint. The left leg is an exoskeleton mechanical leg worn on the healthy leg of the amputees. The right leg is an assisted lower limb prosthesis composed of intelligent lower limb prosthesis and exoskeleton mechanical leg, which is connected to the stump of the disabled thigh through the prosthetic receiving cavity. The assisted lower limb prosthesis is designed as 
detachable components. Intelligent lower limb prosthesis and exoskeleton mechanical legs achieve mechanical integration through the upper knee connector. In the assisted walking mode, the knee joint of assisted lower limb prosthesis is actively driven by electric motor. In the daily walking mode, the assisted lower limb prosthesis can be used alone as the intelligent lower limb prosthesis after the exoskeleton structure is removed, and it is semi-active driven by the magnetorheological damper. The thigh and calf length can be adjusted to suit different groups of people.

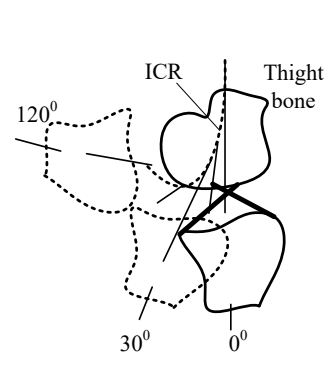

a) ICR of human knee joint Figure 2 ICR of human knee joint and four-bar bionic knee joint

As shown in Fig. 2, the knee movement of human is rather complicated, involving both rolling and sliding. The curve of the instantaneous center of rotation (ICR) of human knee joint is similar to J-shaped curve. According to the principle of knee movement, the four-link bionic knee exoskeleton was designed, such that the exoskeleton has the same center of instantaneous rotation as the actual knee [17].

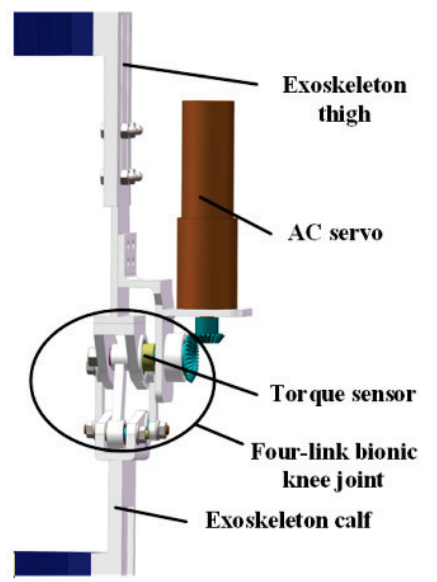

Figure 3 The structure of the exoskeleton mechanical leg with the four-link bionic knee

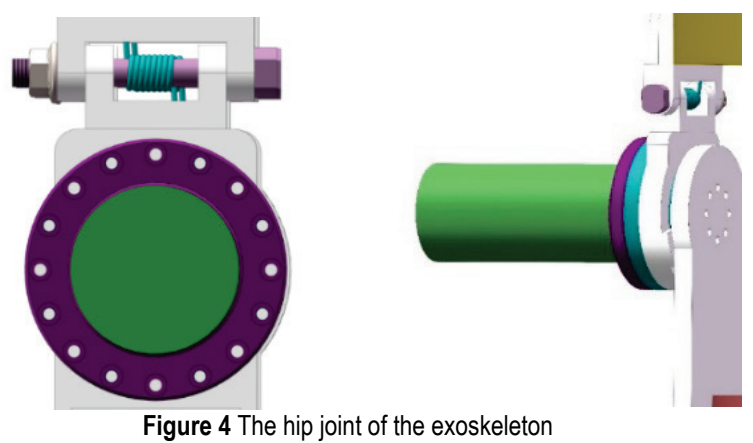

The structure of exoskeleton mechanical leg with the four-link bionic knee is illustrated in Fig. 3. It can be seen that the exoskeleton is powered by a servo motor, while speed reduction and torque amplification are realized by harmonic reducer. For real-time detection of torque, the torque sensor is installed on the driving shaft of the knee. As shown in Fig. 4, the hip joint was designed for flexion, extension, adduction and abduction with two DOFs.

\section{ADMITTANCE CONTROL SYSTEM}

Derived from mechanical impedance, the admittance theory is an equivalent network idea based on generalized inertia, damping and stiffness. In this theory, the relationship between force and response speed is constructed by comparing the actual physical system to an input and output system with admittance (impedance) features [18].

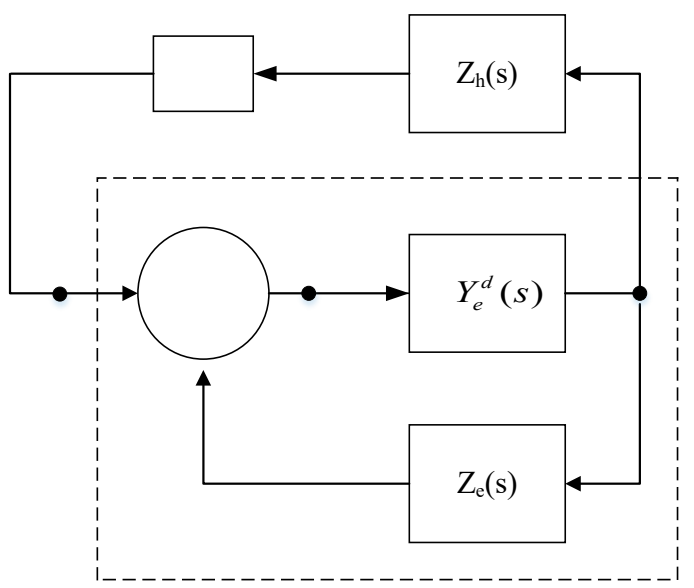

Figure $\mathbf{5}$ The operator-tool model ( $p$ : operator-tool coupling point; $s$ : torque detection point on the driving shaft)

Fig. 5 shows the simplified operator-tool model of the operator's legs and the mechanical legs of the exoskeleton. Under the admittance controller, the mechanical leg moves according to the virtual admittance model below:

$\bar{Y}_{\mathrm{e}}^{\mathrm{d}}=\frac{s}{\bar{I}_{\mathrm{e}}^{\mathrm{d}} s^{2}+\bar{b}_{\mathrm{e}}^{\mathrm{d}} s+\bar{k}_{\mathrm{e}}^{\mathrm{d}}}$

where, $\bar{I}_{\mathrm{e}}^{\mathrm{d}}$ is the inertia moment; $\bar{b}_{\mathrm{e}}^{\mathrm{d}}$ is the damping coefficient; $\bar{k}_{\mathrm{e}}^{\mathrm{d}}$ is the stiffness coefficient. The impedance of the exoskeleton can be expressed as:

$Z_{\mathrm{e}}(s)=\frac{I_{\mathrm{e}} s^{2}+b_{\mathrm{e}} s+k_{\mathrm{e}}}{s}$

The admittance control system of the four-link bionic knee exoskeleton is illustrated in Fig. 6. Obviously, the system encompasses an admittance controller and an inertial compensator. The former is further composed of an admittance model and a linear quadratic (LQ) trajectory tracking controller. 


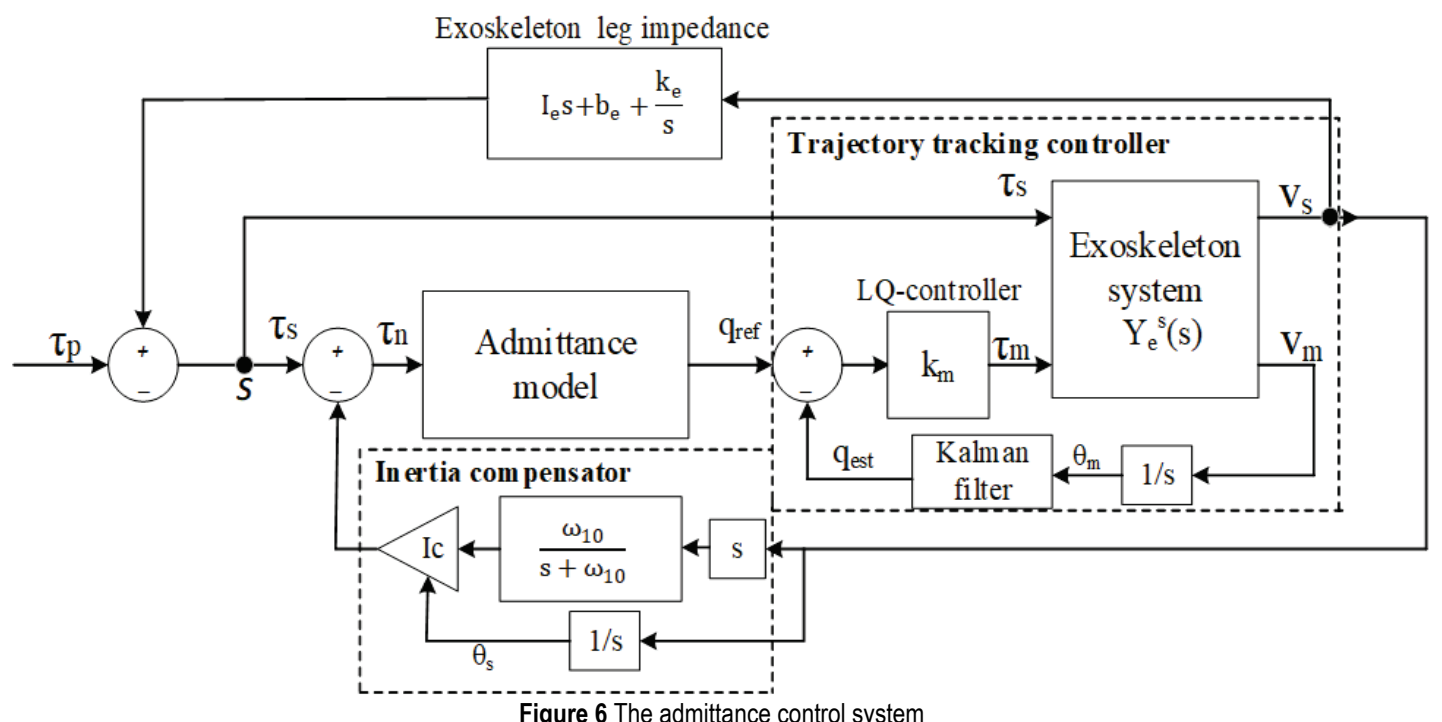

In the state space, the admittance model in Eq. (1) can be expressed as:

$$
\left[\begin{array}{c}
\dot{\theta} \\
\dot{\theta} \\
\dot{\xi}
\end{array}\right]=\left[\begin{array}{ccc}
0 & 1 & 0 \\
-\frac{\bar{k}_{\mathrm{e}}^{\mathrm{d}}}{\bar{I}_{\mathrm{e}}^{\mathrm{d}}} & -\frac{\overline{\mathrm{e}}_{\mathrm{e}}^{\mathrm{d}}}{\bar{I}_{\mathrm{e}}^{\mathrm{d}}} & 0 \\
1 & 0 & 0
\end{array}\right]\left[\begin{array}{c}
\theta \\
\dot{\theta} \\
\xi
\end{array}\right]+\left[\begin{array}{c}
0 \\
\frac{1}{\bar{I}_{\mathrm{e}}^{\mathrm{d}}} \\
0
\end{array}\right] \tau_{\mathrm{n}}
$$

where, $\theta$ is the angle of the mechanical leg relative to the vertical direction; $\xi$ is the angle integral used to reduce the steady-state tracking error; $\tau_{\mathrm{n}}$ is the input of the admittance model, i.e. the sum of the torque $\tau_{\mathrm{s}}$ detected by the sensor and the feedback torque from the inertia compensator. The above control system can be described as:

$$
\dot{q}=\bar{F}_{\mathrm{e}}^{\mathrm{d}} \boldsymbol{q}+\bar{G}_{\mathrm{e}}^{\mathrm{d}} \tau_{\mathrm{n}}
$$

where, $\boldsymbol{q}$ is the state space vector $\boldsymbol{q}=\left[\begin{array}{lll}\theta & \dot{\theta} & \xi\end{array}\right]^{\mathrm{T}}$.

In the control process, the reference trajectory $q_{\text {ref }}(t)$ is generated by numerical integration, and a closed loop system is formed with the LQ controller. In addition, the motion information can be derived from the motor rotation angle measured by the emulated encoder, while the state trajectory can be estimated by the state observer composed of the Kalman filter. The coefficient of determination $R^{2}$ was introduced to evaluate the fidelity of trajectory tracking [19]. For the $2 \mathrm{~Hz}$ sine wave tracking, the fidelity was estimated as $99.3 \%$, indicating that the controller can track the motion of the mechanical leg in an accurate manner.

\section{ADMITTANCE CONTROLLER AND INERTIA COMPENSATION}

\subsection{Admittance Controller}

The admittance controller needs to cover the original admittance (impedance) features of the mechanical leg [20]. Let $k_{\mathrm{e}}$ and $b_{\mathrm{e}}$ be the gravity effect and the damping effect, respectively. The two effects can be eliminated by making $k_{\mathrm{d}}=-k_{\mathrm{e}}$ and $b_{\mathrm{e}}=-b_{\mathrm{e}}$.
Fig. 7 presents the simplified operator-tool coupling model, in which the mechanical leg is driven by the output shaft of the motor via the gear. In the figure, $I_{\mathrm{m}}, I_{\mathrm{s}}$ and $I_{\mathrm{e}}$ are the inertia moments of the output shaft of the motor, the exoskeleton driving system, and between the gear and the torque sensor, respectively. The $I_{\mathrm{m}}$ and $I_{\mathrm{s}}$ are coupled by a spring with the elasticity coefficient of $k_{\mathrm{c}}$ and a damper with the damping coefficient of $b_{\mathrm{c}}$. The torque sensor is installed at point $\mathrm{s}$, the coupling point between $I_{\mathrm{s}}$ and the inertia moments of the mechanical and human legs. Moreover, $\tau_{\mathrm{h}}$ is the kinematic torque of human leg, $\tau_{\mathrm{m}}$ is the driving torque of exoskeleton, and $\tau_{\mathrm{s}}$ is the torque measured by torque sensor; $\theta_{\mathrm{m}}$ and $\theta_{\mathrm{s}}$ are respectively the output shaft angle of the motor and the output angle of the mechanical leg.

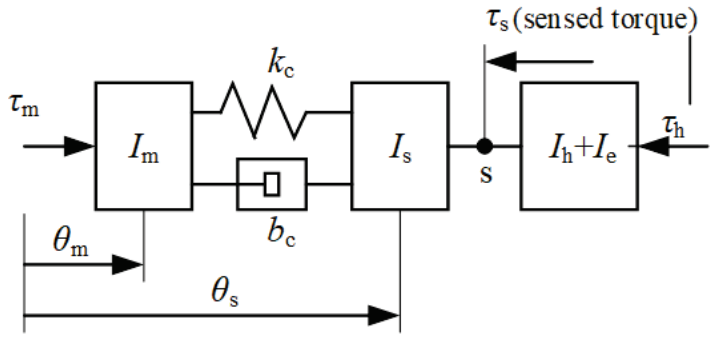

Figure 7 The simplified operator-tool coupling model

In light of Fig. 7, the linear motion equation of the exoskeleton driving system can be expressed as:

$$
\begin{gathered}
\tau_{\mathrm{m}}-k_{\mathrm{c}}\left(\theta_{\mathrm{s}}-\theta_{\mathrm{m}}\right)-b_{\mathrm{c}}\left(v_{\mathrm{s}}-v_{\mathrm{m}}\right)=I_{\mathrm{m}} s v_{\mathrm{m}} \\
\tau_{\mathrm{s}}-k_{\mathrm{c}}\left(\theta_{\mathrm{m}}-\theta_{\mathrm{s}}\right)-b_{\mathrm{c}}\left(v_{\mathrm{m}}-v_{\mathrm{s}}\right)=I_{\mathrm{s}} s v_{\mathrm{s}}
\end{gathered}
$$

The relationship between the input torque and the output speed of the mechanical leg can be described as:

$$
\left[\begin{array}{c}
V_{\mathrm{s}}(s) \\
V_{\mathrm{m}}(m)
\end{array}\right]=Y_{\mathrm{e}}(s)\left[\begin{array}{c}
\tau_{\mathrm{s}} \\
\tau_{\mathrm{m}}
\end{array}\right]\left[\begin{array}{cc}
Y_{\mathrm{e}}^{11} & Y_{\mathrm{e}}^{12} \\
Y_{\mathrm{e}}^{21} & Y_{\mathrm{e}}^{22}
\end{array}\right]\left[\begin{array}{c}
\tau_{\mathrm{s}} \\
\tau_{\mathrm{m}}
\end{array}\right]
$$

The admittance model of the driving system can be derived as: 
$\bar{Y}_{\mathrm{e}}^{\mathrm{d}}=\frac{s}{\bar{I}_{\mathrm{e}}^{\mathrm{d}} s^{2}+\bar{b}_{\mathrm{e}}^{\mathrm{d}} s+\bar{k}_{\mathrm{e}}^{\mathrm{d}}}$

The proportional control law for speed tracking can be written as:

$\tau_{\mathrm{m}}=k_{\mathrm{p}}\left(V_{\mathrm{ref}}-V_{\mathrm{m}}\right)=k_{\mathrm{p}}\left(\bar{Y}_{\mathrm{e}}^{\mathrm{d}}-V_{\mathrm{m}}\right)$

In closed-loop control, the admittance model of the driving system can be depicted as:

$$
Y_{\mathrm{e}}^{\mathrm{s}}=\frac{V_{\mathrm{s}}(s)}{\tau_{\mathrm{s}}(s)}=\frac{s^{3}+\frac{k_{\mathrm{p}}}{I_{\mathrm{m}}} s^{2}+\frac{k_{\mathrm{c}}}{I_{\mathrm{m}}} s+\frac{k_{\mathrm{p}} k_{\mathrm{c}}}{\bar{I}_{\mathrm{e}}^{\mathrm{d}} I_{\mathrm{m}}}}{I_{\mathrm{s}} s^{4}+\frac{I_{\mathrm{s}} k_{\mathrm{p}}}{I_{\mathrm{m}}} s^{3}+\frac{k_{\mathrm{c}}\left(I_{\mathrm{s}}+I_{\mathrm{m}}\right)}{I_{\mathrm{m}}} s^{2}+\frac{k_{\mathrm{c}} k_{\mathrm{p}}}{I_{\mathrm{m}}} s}
$$

The minimum admittance controller with inertia compensation is presented in Fig. 8, where the load $\left(I_{\mathrm{h}}+I_{\mathrm{e}}\right)$ is the total load of mechanical and human legs; $H_{\mathrm{i}}(s)$ is the inertial compensator.

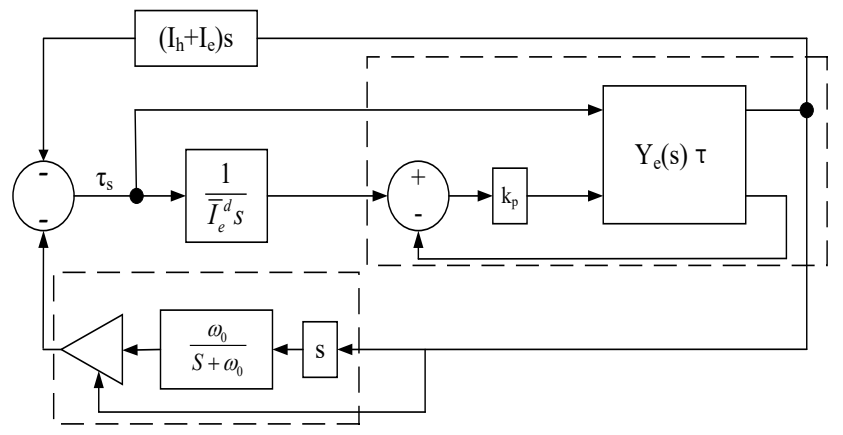

Figure 8 Simplified admittance controller with inertia compensation

The load acting on the driving system can be computed as:

$Z_{\text {load }}=\left(I_{\mathrm{e}}+I_{\mathrm{h}}\right) s$

The admittance of the muscles' toque $\tau_{\mathrm{h}}$ under the coupling between mechanical and human legs can be given by:

$$
Y_{\mathrm{e}}^{\mathrm{h}}(s)=\frac{V_{\mathrm{s}}(s)}{\tau_{\mathrm{h}}(s)}=\frac{Y_{\mathrm{e}}^{\mathrm{s}}(s)}{1+\left[H_{\mathrm{i}}(s)+Z_{\text {load }}(s)\right] Y_{\mathrm{e}}^{\mathrm{s}}(s)}
$$

\subsection{INERTIA COMPENSATION}

After wearing exoskeleton, the operator will be less flexible in leg movement, owing to the weight of the exoskeleton, the friction and inertia [21]. To ensure the flexibility, the exoskeleton weight and friction can be compensated by the controller, while the inertia can only be compensated for by proper parameter setting.

To begin with, the angular acceleration should be obtained from the differential angular speed of the mechanical leg, and the cut off frequency be controlled to the normal swing frequency of human leg by the low-pass filter and multiplied by a gain coefficient $\omega_{0}$. The transfer function of analog inertial compensator can be defined as:

$H_{\mathrm{i}}(s)=\frac{I_{\mathrm{c}} \omega_{0} s}{s+\omega_{0}}$

The admittance model is employed to offset the damping and weight of the exoskeleton. When $I_{\mathrm{c}}=0$, the inertia felt by the operator equals the total inertia of the mechanical leg and the driving system. In theory, the total inertia moment of the tool should be offset before the operator's inertia moment:

$I_{\mathrm{c}}=(\alpha-1) I_{\mathrm{h}}-I_{\mathrm{e}} \quad(0<\alpha<1)$

The linear second-order impedance models of the human leg and the mechanical leg can be respectively defined as:

$Z_{\mathrm{h}}(s)=\frac{I_{\mathrm{h}} s^{2}+b_{\mathrm{h}} s+k_{\mathrm{h}}}{s}$

$Z_{\mathrm{e}}(s)=\frac{I_{\mathrm{e}} s^{2}+b_{\mathrm{e}} s+k_{\mathrm{e}}}{s}$

In our four-link bionic knee exoskeleton, the bionic knee can simulate the movement of the human knee accurately with its special structure. Since the inertial moment of the calf changes instantaneously with the knee, the inertia compensation should restore the original inertia precisely. Thus, it is necessary to obtain the change of the inertia of the entire calf with the knee angle.

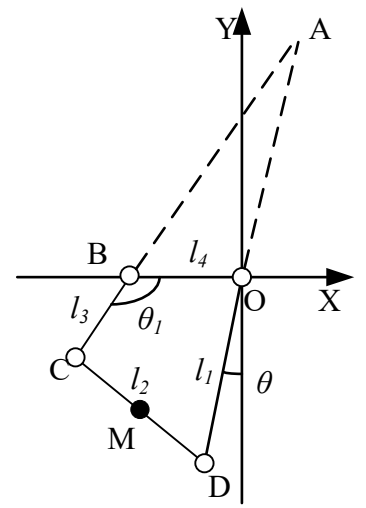

Figure 9 Simplified structure of four-link knee

To acquire the equivalent inertia moment of four-link bionic knee, the four-link knee structure was simplified (Fig. 9), and examined with the following theories on the kinetic energy of particle system, the kinematic energy of rigid body around rotating shaft, and the plane motion of rigid body [22-24]. In this way, the inertia moment of the 1DOF planar four-link system can be obtained equivalent to the prime mover. The equivalent inertia moment of the four-link bionic knee can be expressed as:

$I_{\mathrm{e}}=J_{\mathrm{v}}=J_{1}+\left(2 \cdot J_{2}+m_{2} \cdot O M^{2}\right) \cdot \frac{L_{1}^{2}}{O A^{2}}+J_{3} \cdot \frac{L_{1}^{2} \cdot O B^{2}}{L_{3}^{2} \cdot O A^{2}}$ 
where, $J_{1}$ is the inertia moment of link $O D$ to point $O ; J_{2}$ is the inertia moment of link $C D$ to mass center $M ; J_{3}$ is the inertia moment of link $B C$ to point $B$.

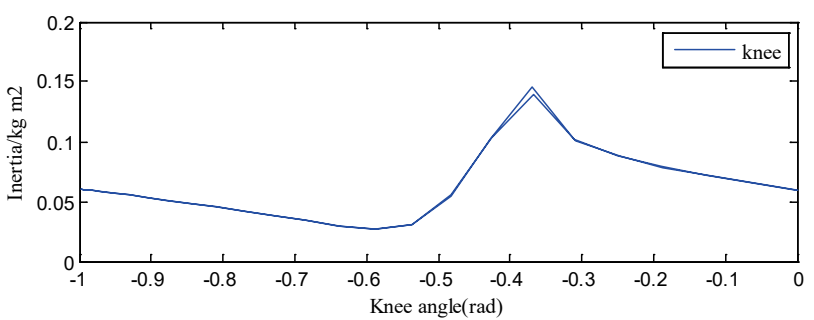

Figure 10 Inertia moment variation of mechanical leg

Fig. 10 shows how the inertia moment of the mechanical leg changes as the knee rotates within $-1^{\circ}$ around its driving shaft. Obviously, the inertia moment of the mechanical leg always changes instantaneously with the knee angle, and peaks at $0.146 \mathrm{kgm}^{2}$ when the angle of the driving shaft of the knee is $0.37^{\circ}$.

\section{SIMULATION VERIFICATION}

To validate the admittance control algorithm, i.e. admittance control with inertia compensation, the human leg parameters of an operator (height: $175 \mathrm{~cm}$; weight: 70 $\mathrm{kg}$ ) were computed by the method in the previous research $[25,26]: I_{\mathrm{h}}=0.26 \mathrm{kgm}^{2}, b_{\mathrm{h}}=2.0 \mathrm{mms} /{ }^{\circ}$ and $k_{\mathrm{h}}=11.0 \mathrm{Nm} /{ }^{\circ}$. The parameters of the driving mechanism are $I_{\mathrm{e}}=0.185$ $\mathrm{kgm}^{2}, I_{\mathrm{m}}=0.006 \mathrm{kgm}^{2}$ and $I_{\mathrm{s}}=0.0091 \mathrm{kgm}^{2}$. The parameters of the exoskeleton are $w_{0}=24 \% \mathrm{~s}, g=9.8 \mathrm{~m} / \mathrm{s}^{2}$ and $k=3000 \mathrm{~N} / \mathrm{m}$ (elastic modulus).

It is assumed that the operator is walking at a uniform speed $(1 \mathrm{~m} / \mathrm{s})$. Our simulation only considers the motion of the swinging leg (the other leg stands still as the support). Then, the leg movement can be simplified as pendulum motion, similar to sinusoidal motion. During the simulation, the operator's swinging leg moved in a sinusoidal manner at different amplitudes and frequencies. For simplicity, the initial state of the leg was considered as: the thigh is perpendicular to the calf. Three simulations were set up, respectively with the desired knee trajectory of $\theta_{1}=0.1744 \sin (t), \theta_{1}=0.3489 \sin (t)$ and $\theta_{1}=0.5234 \sin (t)$.

The simulation results of the proposed control method on the four-link biometric knee exoskeleton are listed in Figs. 11 and 12.

Fig. 11 shows that the four-link bionic knee exoskeleton was adjusted rapidly at the initial stage of motion and achieved a tracking error of less than $5 \%$, whichever the amplitude of the motion trajectory. As can be seen from Fig. 12, the tracking error was $\pm 0.5^{\circ}, \pm 1.0^{\circ}$ and $\pm 1.5^{\circ}$ at the motion trajectory amplitude of $10^{\circ}, 20^{\circ}$ and $30^{\circ}$, respectively, when the amplitude remained in the stable stage $(5 \sim 10 \mathrm{~s})$. Hence, the proposed control method, i.e. admittance control with inertia compensation, enables the four-link bionic knee exoskeleton to track the motion of operator's knee in an accurate manner.

For an operator wearing the bionic knee exoskeleton, the upper body and the lower body may be affected differently. The operator-tool interaction can partially reflect the quality of exoskeleton control. To measure the interaction, a bandage was used to bind the bionic knee exoskeleton to the operator, and the interaction force was simulated on ADAMs. The simulated force was referenced to analyze the impedance of the exoskeleton to the operator.

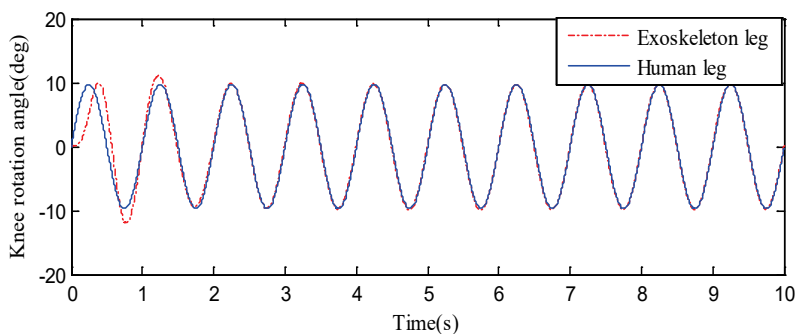

(a) The tracking curve at the amplitude of $10^{\circ}$

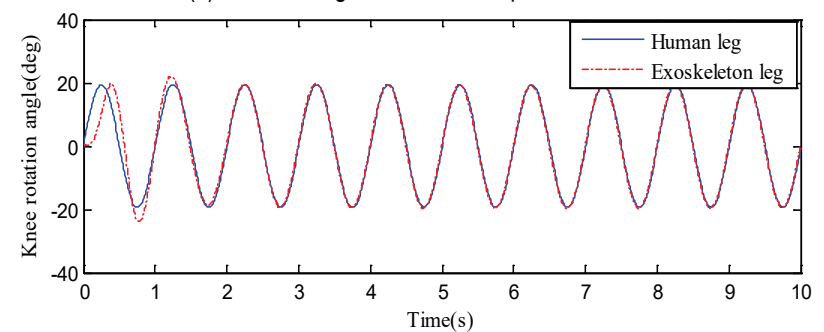

(b) The tracking curve at the amplitude of $20^{\circ}$

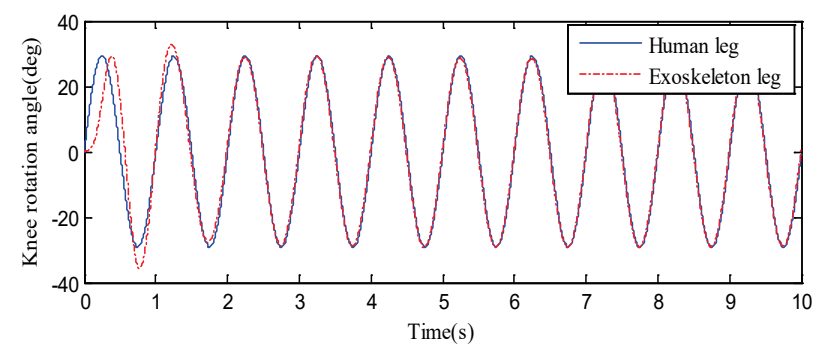

(c) The tracking curve at the amplitude of $30^{\circ}$ Figure 11 Tracking curve of the exoskeleton

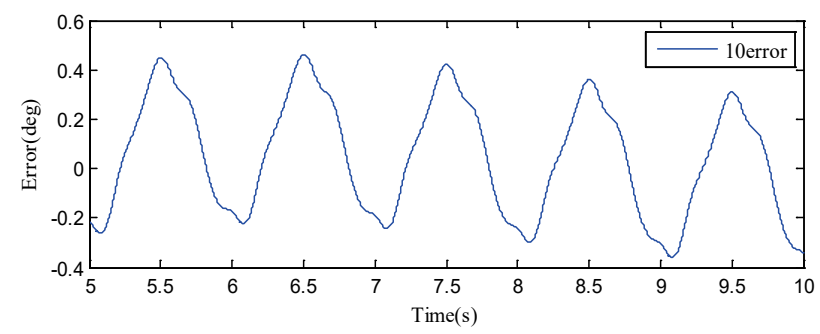

(a) The tracking error at the amplitude of $10^{\circ}$

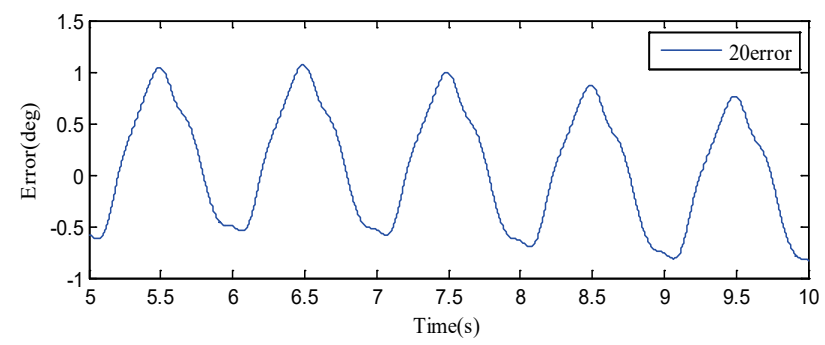

(b) The tracking error at the amplitude of $20^{\circ}$

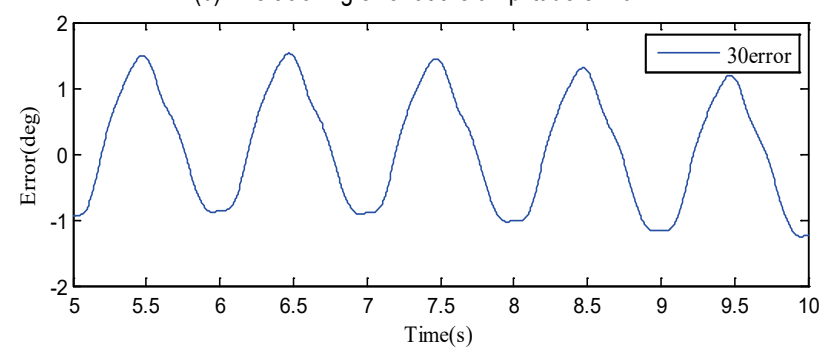

(c) The tracking error at the amplitude of $30^{\circ}$

Figure 12 Tracking error of the exoskeleton 
As shown in Fig. 13, the interaction force between the exoskeleton and the operator was about $6 \mathrm{~N}$. Under the admittance control with inertia compensation, the interaction force changed differently with motion amplitudes. The peak interaction force $(0.5 \mathrm{~N})$ at the amplitude of $30^{\circ}$ was greater than that at $10^{\circ}$ or $20^{\circ}$. The value was $91.7 \%$ lower than that of the operator without wearing the four-link bionic knee exoskeleton. This means the proposed control method can indeed reduce the impedance of the exoskeleton to the human body, and ensure the tracking of the tool to operator motion with a little contact force [27, 28].

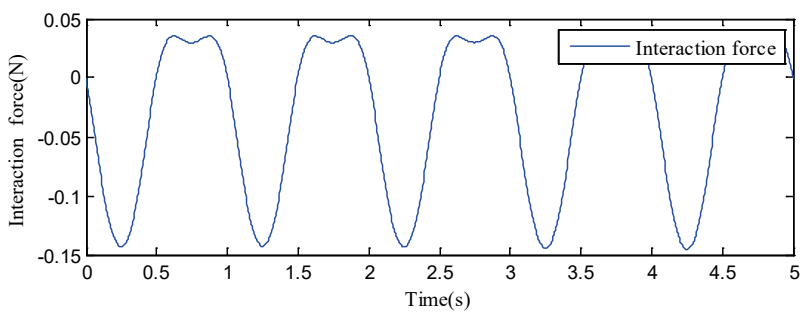

(a) The interaction force curve at the amplitude of $10^{\circ}$

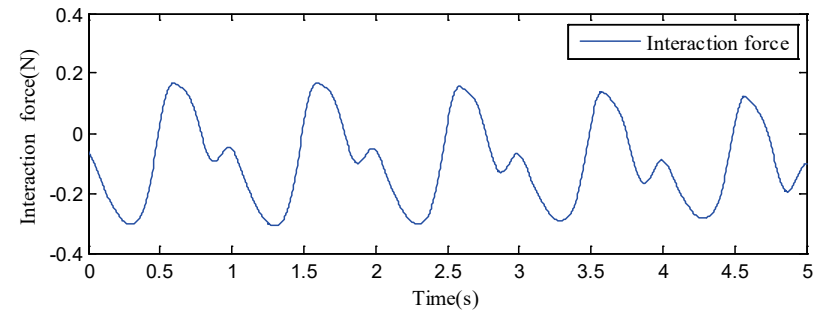

(b) The interaction force curve at the amplitude of $20^{\circ}$

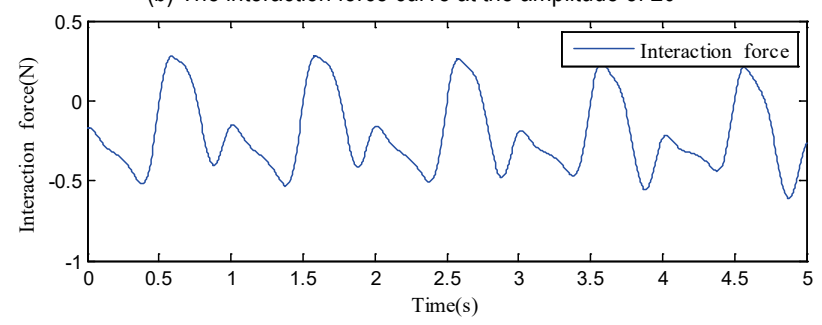

(c) The interaction force curve at the amplitude of $30^{\circ}$ Figure 13 The interaction force curve

\section{CONCLUSIONS}

This paper puts forward the admittance control method with inertia compensation, and verifies its effect in controlling the swinging tracking of four-link bionic knee exoskeleton. Since the calf inertia changes continuously with the rotation of the knee, the inertia of the mechanical leg was compensated by the equivalent method, i.e. setting up the inertia curve of the mechanical leg tracking the change of knee angle. The proposed control method was proved through simulation. The simulation results confirm that our method ensures the tracking of the exoskeleton of operator motion, coordinate operator-tool motions, and reduces the interaction force.

To sum up, admittance model for the four-link bionic knee exoskeleton can reflect the real motion behavior and identify the human intention. The admittance control with inertia compensation can control the complex bionic knee exoskeleton well, allowing the latter to track the operator motion in an accurate manner. The proposed control method can also restore the original admittance features of the exoskeleton, and reduce the impedance of the exoskeleton to the human body.

Of course, the maximum tracking error is relatively high, owing to the complex and flexible structure of the four-link bionic knee exoskeleton. The error will be further reduced in future research through motion reversing. Besides, a wearable four-link bionic knee exoskeleton will be produced for experimental verification at different frequencies and faster swinging, aiming to disclose the actual impact of our method on the exoskeleton.

\section{Acknowledgements}

Financial supports from the Fundamental Research Funds for the Central Universities (ID N170313025) and Scientific Study Project of Liaoning Province Education Department (ID LQGD2017029) are highly appreciated.

\section{REFERENCES}

[1] Liu, D. F., Tang, Z. Z., \& Pei, Z. C. (2015). Lower extremity exoskeleton swing control based on admittance principle. Journal of Beijing University of Aeronautics and Astronautics, 41(6), 1019-1025.

[2] Gupta, A., Mondal, A. K., Gupta, M. K. (2019). Kinematic, dynamic analysis and control of 3 DOF upper-limb robotic exoskeleton. Journal Européen des Systèmes Automatisés, 52(3), 515-520. https://doi.org/10.18280/jesa.520311

[3] Beyl, P. V., Damme, M., Ham, R. V., Vanderborght, B., \& Lefeber, D. (2014). Design and control of a lower limb exoskeleton for robot-assisted gait training. Applied Bionics \& Biomechanics, 6(2), 229-243. https://doi.org/10.1155/2009/580734

[4] Huo, W., Mohammed, S., \& Amirat, Y. (2019). Impedance Reduction Control of a Knee Joint Human-Exoskeleton System. IEEE Transactions on Control Systems Technology, 27(6), 2541-2556. https://doi.org/10.1109/TCST.2018.2865768

[5] Moon, D. H., Kim, D., \& Hong, Y. D. (2019). Development of a single leg knee exoskeleton and sensing knee center of rotation change for intention detection. Sensors (Switzerland), 19(18), 3960. https://doi.org/10.3390/s19183960

[6] Yu, S., Huang, T. H., Wang, D., Lynn, B., Sayd, D., Silivanov, V., Park, Y. S., Tian, Y., \& Su, H. (2019). Design and Control of a High-Torque and Highly Backdrivable Hybrid Soft Exoskeleton for Knee Injury Prevention during Squatting. IEEE Robotics and Automation Letters, 4(4), 4579-4586. https://doi.org/10.1109/LRA.2019.2931427

[7] Xie, H. L., Liu, Z. B., \& Yang, J. Y. (2016). Modelling of magnetorheological damper for intelligent bionic Leg and simulation of knee joint movement control. International Journal of Simulation Modelling, 15(1), 144-156. https://doi.org/10.2507/IJSIMM15(1)CO2

[8] Hamon, A., Aoustin, Y., \& Caro, S. (2014). Two walking gaits for a planar bipedal robot equipped with a four-bar mechanism for the knee joint. Multibody System Dynamics, 3(1), 283-307. https://doi.org/10.1007/s11044-013-9382-7

[9] Kazerooni, H., Racine, J. L., Huang, L., \& Steger, R. (2006). On the Control of the Berkeley Lower Extremity Exoskeleton (BLEEX). IEEE International Conference on Robotics and Automation, 4345-4352. https://doi.org/10.1177/0278364906065505

[10] Kazerooni, H., Steger, R., \& Huang, L. H. (2006). Hybrid control of the berkeley lower extremity exoskeleton (BLEEX). IEEE/ASME Transactions on Mechatronics, 25(5-6), 561-573. https://doi.org/10.1177/0278364906065505

[11] Kawamoto, H., Lee, S., Kanbe, S., \& Sankai, Y. (2003). Power assist method for HAL-3 using EMG-based feedback 
controller. IEEE International Conference on Systems, Man and Cybernetics, 1648-1653. https://doi.org/10.1109/ICSMC.2003.1244649

[12] Kawamoto, H., Hayashi, T., Sakurai, T., Eguchi, K., \& Sankai, Y. (2009). Development of single leg version of HAL for hemiplegia. International Conference of the IEEE Engineering in Medicine \& Biology Society, 2009, 50385043. https://doi.org/10.1109/IEMBS.2009.5333698

[13] Aguirre-Ollinger, G., Colgate, J. E., Peshkin, M. A., \& Goswami, A. (2011). Design of an active one-degree-offreedom lower-limb exoskeleton with inertia compensation. International Journal of Robotics Research, 30(4), 486-499. https://doi.org/10.1177/0278364910385730

[14] Aguirre-Ollinger, G., Colgate, J. E., Peshkin, M. A., \& Goswami, A. (2009). Active-Impedance Control of a LowerLimb Assistive Exoskeleton. IEEE, International Conference on Rehabilitation Robotics, 188-195.

[15] Breakey, J. W. (1998). Theory of integrated balance: the lower limb amputee. JPO Journal of Prosthetics \& Orthotics, 10(2), 42-44. https://doi.org/10.1097/00008526-199801020-00006

[16] Kordasz, M. \& Sauer, P. (2013).Automatic determination of knee kinematics for lower limb rehabilitation manipulator design. Robot Motion \& Control. https://doi.org/10.1109/RoMoCo.2013.6614589

[17] Xiao, B., Shao, Y., \& Zhang, W. (2019). Design and optimization of single-degree-of-freedom six- bar mechanisms for knee joint of lower extremity exoskeleton robot. IEEE International Conference on Robotics and Biomimetics, ROBIO 2019, 2861-2866. https://doi.org/10.1109/ROBIO49542.2019.8961400

[18] Yang, Z. (2005). Research on the flexibility control method of robot based on impedance control. Doctoral dissertation, Southeast University.

[19] Nagarajan, U., Aguirre-Ollinger, G., \& Goswami, A. (2015) Integral Admittance Shaping for exoskeleton control. IEEE International Conference on Robotics and Automation, 2015, 5641-5648. https://doi.org/10.1109/ICRA.2015.7139989

[20] Aguirre-Ollinger, G., Nagarajan, U., \& Goswami, A. (2016). An admittance shaping controller for exoskeleton assistance of the lower extremities. Autonomous Robots, 40(4), $701-$ 728. https://doi.org/10.1007/s10514-015-9490-8

[21] Aguirre-Ollinger, G., Colgate, J. E., Peshkin, M. A., \& Goswami, A. (2007). A 1-DOF assistive exoskeleton with virtual negative damping: effects on the kinematic response of the lower limbs. IEEE/RSJ International Conference on Intelligent Robots and Systems, 1938-1944. https://doi.org/10.1109/IROS.2007.4399147

[22] Zhang, Y.Q. \& Xu, S. T. (2006). Calculation formula and application of equivalent rotational inertia of plane four connecting rod mechanism. One heavy technique, 3, 15-17.

[23] Qu, X. Q., Jiao, Y. H., Chen, Z. B., \& Kan, L. H. (2004). Formula for calculating the equivalent moment of inertia of a planar linkage with single degree of freedom. Journal of Harbin Institute of Technology, 36(5), 610-612.

[24] Chen, Z., Lei, J., Cheng, L., \& Gao, T. (2019). Hopping planning of the bionic leg mechanism driven by PAMs with biarticular muscle, High Technology Letters, 25(4), 408-416.

[25] Winter, D. A. (2009). Biomechanics and motor control of human movement / d.a. winter. Physiotherapy, 74(2), 94-94. https://doi.org/10.1002/9780470549148

[26] Winter, D. A. (1984). Biomechanics of human movement with applications to the study of human locomotion. Critical Reviews in Biomedical Engineering, 9(4), 287-314.

[27] He, Y. T. (2008). Research on position based gas and liquid servo impedance control system. Doctoral dissertation, Harbin Institute of Technology.

[28] Aguirre-Ollinger, G. (2015). Exoskeleton control for lowerextremity assistance based on adaptive frequency oscillators: adaptation of muscle activation and movement frequency.
Proceedings of the Institution of Mechanical Engineers Part H Journal of Engineering in Medicine, 229(1), 52-68. https://doi.org/10.1177/0954411914567213

\section{Contact information:}

Fei LI, PhD, Lecturer

School of Information Science and Engineering,

Shenyang University of Technology,

Shenyang 110870, China

E-mail: lifeisut@163.com

\section{Qingdong WANG}

School of Mechanical Engineering and Automation, Northeastern University,

Shenyang 110819, China

E-mail: wqd1221@163.com

Yao XIE

School of Mechanical Engineering and Automation,

Northeastern University,

Shenyang 110819, China

E-mail:1870220@stu.neu.edu.cn

Hualong $\mathrm{XIE}, \mathrm{PhD}$, associate professor

(Corresponding author)

School of Mechanical Engineering and Automation,

Northeastern University,

Shenyang 110819, China

E-mail: hlxie@mail.neu.edu.cn 